\title{
Redox regulation of mitochondrial functional activity by quinones
}

\author{
NG Krylova ${ }^{1}$, TA Kulahava ${ }^{1}$, VT Cheschevik ${ }^{2}$, IK Dremza ${ }^{2}$, GN Semenkova ${ }^{3}$, \\ IB Zavodnik ${ }^{2}$
}

\author{
${ }^{1}$ Department of Biophysics, Faculty of Physics, Belarusian State University, Minsk, Belarus \\ ${ }^{2}$ Department of Biochemistry, Yanka Kupala State University of Grodno, Grodno, Belarus \\ ${ }^{3}$ Department of Radiation Chemistry and Pharmaceutical Technologies, Faculty of Chemistry, \\ Belarusian State University, Minsk, Belarus
}

Received: October 29, 2015

Accepted: October 8, 2016

\begin{abstract}
Quinones are among the rare compounds successfully used as therapeutic agents to correct mitochondrial diseases and as specific regulators of mitochondrial function within cells. The aim of the present study was to elucidate the redox-dependent effects of quinones on mitochondrial function. The functional parameters [respiratory activity, membrane potential, and reactive oxygen species (ROS) generation] of isolated rat liver mitochondria and mitochondria in intact cells were measured in the presence of eight exogenously applied quinones that differ in lipophilicity and one-electron reduction potential. The quinones affected the respiratory parameters of mitochondria, and dissipated the mitochondrial membrane potential as well as influenced (either decreased or enhanced) ROS generation, and restored the electron flow during electron transport chain inhibition. The stimulation of ROS production by juglone and 2,5-di-tert-butyl-1,4-benzoquinone was accompanied by a decrease in the acceptor control and respiration control ratios, dissipation of the mitochondrial membrane potential and induction of the reverse electron flow under succinate oxidation in isolated mitochondria. Menadione and 2,3,5-trimethyl-1,4-benzoquinone, which decreased the mitochondrial ROS generation, did not affect the mitochondrial potential and, vice versa, were capable of restoring electron transport during Complex I inhibition. In intact C6 cells, all the quinones, except for coenzyme $\mathrm{Q}_{10}$, decreased the mitochondrial membrane potential. Juglone, 1,4-benzoquinone, and menadione showed the most pronounced effects. These findings indicate that quinones with the reduction potential values $E_{1 / 2}$ in the range from -99 to $-260 \mathrm{mV}$ were effective redox regulators of mitochondrial electron transport.

Keywords: quinone, mitochondria, respiration, membrane potential, reactive oxygen species, one-electron reduction potential
\end{abstract}

\section{Introduction}

Quinones are oxidized derivatives of aromatic compounds (such as benzene or naphthalene) stabilized by conjugation (Fig. 1). Electron-donating substituents, such as phenols and catechols, increase the nucleophilicity of the ring and contribute to high redox potential of the quinones. Some quinones serve as electron acceptors in electron transport chains (ETCs), such as those participating in photosynthesis (plastoquinone, phylloquinone), and aerobic respiration (ubiquinone). Quinones are capable of regulating cell functions, inducing reactive oxygen species (ROS) generation or, vice versa, manifesting antioxidant properties (in a reduced form). Another mechanism for quinone regulation of cellular properties is arylation

Corresponding author: Ilya B Zavodnik, PhD, DSci

Department of Biochemistry, Yanka Kupala State University of Grodno

Bulvar Leninskogo Komsomola 50, 230030 Grodno, Belarus

Phone: +375 152 484583; Fax: +375 152 434121; E-mail: zavodnik_i1@mail.ru 
<smiles>O=C1C=CC(=O)C=C1</smiles>

1,4-benzoquinone

(1)<smiles>CC(C)(C)C1=CC(=O)C(C(C)(C)C)=CC1=O</smiles>

2,5-di-tert-butyl1,4-benzoquinone (2)<smiles>CC1=CC(=O)C(C)=C(C)C1=O</smiles>

2,3,5-trimethyl1,4-benzoquinone

(3)<smiles>COC1=C(OC)C(=O)C(C)=CC1=O</smiles>

coenzyme $\mathrm{Q}_{0}$

(4)<smiles></smiles>

coenzyme $\mathrm{Q}_{10}$

(5)<smiles>CC1=CC(=O)c2ccccc2C1=O</smiles>

menadione

(6)<smiles>O=C1C=CC(=O)c2c(O)cccc21</smiles>

juglone

(7)<smiles>O=C1C=C(O)C(=O)c2ccccc21</smiles>

lawsone

(8)
Fig. 1. Structure of the quinones used

of $\mathrm{SH}$ and $\mathrm{NH}_{2}$ groups of some functionally significant proteins $(6,39,49,50,55)$. The rate and direction of oxidation-reduction reactions are determined by quinone reduction potentials. Quinones with one-electron reduction potential $\left(E_{1 / 2}\right)$ values in the range from -240 to $-170 \mathrm{mV}$ and from -50 to $25 \mathrm{mV}$ are effective redox cyclic agents and induce oxidative stress in biological systems $(14,43,50)$. Quinones can be reduced according to one- or twoelectron mechanisms by intracellular reductases, including mitochondrial NADH:ubiquinone oxidoreductase, and the mitochondrial isoform of DT-diaphorase $(\mathrm{NAD}(\mathrm{P}) \mathrm{H}$ : quinone oxidoreductase 1 (NQO1) $(29,38-40)$. Quinones can recapture electrons from ubiquinol of the mitochondrial ETC and influence the electron flow between ETC dehydrogenases (5, 10, 11, 31). Quinone's ability to interact with ETC components depends on the lipophilic properties of a quinone $(5,11,31)$.

Mitochondria are the major cellular sites of ROS production. Earlier studies have suggested that Complexes I (producing $\mathrm{O}_{2}{ }^{--}$to the matrix) and III (producing $\mathrm{O}_{2}{ }^{-}$to both the matrix and intermembrane space) of the mitochondrial ETC are the main sites of the ROS generation (4). Generated by mitochondria, ROS act as redox signals in triggering cellular events such as apoptosis, proliferation, and senescence (35) and quinones, depending on their structure, affect mitochondrial ROS generation. It was shown that 1,4-benzoquinone, 1,4-naphthoquinone, and their derivatives decreased the mitochondrial membrane potential in cells and calcium-loaded isolated mitochondria. This effect is related to mitochondrial permeability transition pore opening caused by quinone-induced ROS generation and/or by arylation of $\mathrm{SH}-$ groups of proteins participating in pore formation $(5,20,21,24,26,41,51)$. Menadione, duroquinone, and coenzyme $\mathrm{Q}_{1}$ are capable of recovering respiratory and phosphorylating mitochondrial functions shunting ETC complexes during rotenone 
Table I. One-electron reduction potential $\left(E_{1 / 2}\right)(7,38)$ and partition coefficient in octanol/water system $(\log P)(53)$ for the quinones studied

\begin{tabular}{|l|l|c|c|}
\hline & \multicolumn{1}{|c|}{ Quinones } & $\log \boldsymbol{P}$ & $\boldsymbol{E}_{\mathbf{1} 2}, \mathbf{m V}$ \\
\hline 1 & BQ & 0.2 & +78 \\
\hline 2 & DTBBQ & 3.4 & -260 \\
\hline 3 & TMBQ & 1.8 & -165 \\
\hline 4 & Coenzyme $\mathrm{Q}_{0}$ & 0.8 & -110 \\
\hline 5 & Coenzyme $\mathrm{Q}_{10}$ & 19.4 & $-230^{\mathrm{a}} ;-36^{\mathrm{b}}$ \\
\hline 6 & Menadione & 2.2 & -203 \\
\hline 7 & Juglone & 1.9 & -99 \\
\hline 8 & Lawsone & 0.9 & -415 \\
\hline
\end{tabular}

${ }^{\mathrm{a}}$ Theoretically calculated value (52); ${ }^{\mathrm{b}}$ experimental value measured for submitochondrial particles from beef-heart mitochondria (15)

inhibition of respiration. Being reduced by mitochondrial DT-diaphorase, these quinones either can transfer electrons directly to Complex III, coenzyme Q, or they can play a role of succinate dehydrogenase substrates $(9,10,13,23,37)$.

Quinone derivatives are among rare compounds successfully used as therapeutic agents to treat mitochondrial diseases $(5,9)$ and bypass the damaged sites in mitochondria. To reveal the dependence of quinone mitochondrial effects on their physicochemical properties, the group of quinones varying in lipophilicity and one-electron reduction potential $E_{1 / 2}$ was studied. We elucidated the effects of eight added quinones: 5-hydroxy-1,4-naphthoquinone (juglone), 1,4-benzoquinone (BQ), 2,5-di-tert-butyl-1,4-benzoquinone (DTBBQ), 2-methyl1,4-naphthoquinone (menadione), 2-hydroxy-1,4-naphthoquinone (lawsone), coenzymes $\mathrm{Q}_{0}$ and $\mathrm{Q}_{10}$, and 2,3,5-trimethyl-1,4-benzoquinone (TMBQ) (Fig. 1) on the respiratory parameters, mitochondrial membrane potential, and ROS production by isolated rat liver mitochondria. The partition coefficients in the octanol/water system $(\log P)$ and $E_{1 / 2}$ of quinones used in this study are represented in Table I.

\section{Materials and Methods}

\section{Chemicals}

Succinic acid disodium salt hexahydrate, L-glutamic acid sodium salt, maleic acid disodium salt, carbonyl cyanide p-(trifluoromethoxy) phenylhydrazone (FCCP), menadione, coenzyme $\mathrm{Q}_{0}$, coenzyme $\mathrm{Q}_{10}$, lawsone, juglone, rotenone, amytal, 3,3'-methylene-bis (4-hydroxycoumarin) (dicumarol), and ADP were purchased from Sigma-Aldrich Chemie $\mathrm{GmbH}$ (Steinheim, Germany), and the fluorescent probes $2^{\prime}, 7^{\prime}$-dichlorodihydrofluorescein diacetate $\left(\mathrm{H}_{2} \mathrm{DCF}-\mathrm{DA}\right), \mathrm{JC}-1$, and MitoSOX Red were purchased from Molecular Probes, Inc. (USA). BQ, TMBQ, and DTBBQ were synthesized and purified, as described by Flaig et al. (19); Fieser and Fieser (18); and Adams et al. (1). All other reagents were purchased from POCh (Gliwice, Poland) and Reakhim (Moscow, Russia) and were of analytical grade. All solutions were made with water purified in the Milli-Q system. 
Quinones were dissolved in dimethyl sulfoxide (DMSO). The final DMSO content in the probe did not exceed $0.1 \%$.

\section{Rat liver mitochondria: Isolation and respiration measurements}

Mitochondria were isolated by the standard procedure of differential centrifugation from the rat liver (27). The liver was quickly removed and placed into the ice-cold isolation medium containing $250 \mathrm{mM}$ sucrose, $20 \mathrm{mM}$ Tris- $\mathrm{HCl}, 1 \mathrm{mM}$ EDTA, $\mathrm{pH}$ 7.2. The tissue was cut into small pieces and homogenized in a glass-Teflon homogenizer with isolation medium (at $2{ }^{\circ} \mathrm{C}$ ). The homogenate was centrifuged at $600 \times g$ for $10 \mathrm{~min}$, and the supernatant was centrifuged at $8,500 \times g$ for $10 \mathrm{~min}$. The obtained pellet was washed in buffer containing $250 \mathrm{mM}$ sucrose, $5 \mathrm{mM}$ Tris- $\mathrm{HCl}$, and $\mathrm{pH} 7.2$ (at $2{ }^{\circ} \mathrm{C}$ ). The mitochondrial pellet was resuspended in the buffer to an approximate protein concentration of $35-40 \mathrm{mg} / \mathrm{ml}$. The protein concentration was determined as described by Lowry et al. (34). Animals were killed according to the rules defined by the European Convention for the Protection of Vertebrate Animals Used for Experimental and Other Scientific Purposes. The protocol was approved by the Bioethics Committee of the Institute for Biochemistry of Biologically Active Compounds of the National Academy of Sciences of Belarus (registration number 4-axd dated March 18, 2014).

The respiration of mitochondria was measured using a laboratory-made oxygen Clarktype electrode and a hermetic polarographic cell (volume $1.25 \mathrm{ml}$ ) with constant gentle stirring (16). The electrode was calibrated by bubbling the polarographic cell with pure nitrogen $\left(\mathrm{pO}_{2}=0\right)$ and atmospheric air (atmospheric $\left.\mathrm{pO}_{2}\right)$. The oxygen consumption by the electrode was negligible. The incubation medium contained $125 \mathrm{mM} \mathrm{KCl}, 50 \mathrm{mM}$ sucrose, $10 \mathrm{mM}$ Tris- $\mathrm{HCl}, 2.5 \mathrm{mM} \mathrm{KH}_{2} \mathrm{PO}_{4}, 5 \mathrm{mM} \mathrm{MgSO}_{4}$, with $0.5 \mathrm{mM}$ EDTA (EDTA containing) or without EDTA (EDTA-free medium), $\mathrm{pH} 7.4$. The experiments were performed at $25^{\circ} \mathrm{C}$ using $5 \mathrm{mM}$ L-glutamate and $2 \mathrm{mM}$ malate mixture $(\mathrm{Glu}+\mathrm{Mal})$ as respiratory substrates. Mitochondrial protein concentration in the sample was $1.0 \mathrm{mg} / \mathrm{ml}$. The functional activity of mitochondria was determined by the acceptor control ratio (ACR), equal to the ratio of the respiratory rates $\left(V_{3} / V_{2}\right)$ of mitochondria in States 3 and 2, the respiration control ratio $\left(\mathrm{RCR}=V_{3} / V_{4}\right)$ and the coefficient of phosphorylation $(\mathrm{ADP} / \mathrm{O})$ (the ratio of the amount of ADP added to the amount of oxygen consumed throughout phosphorylation, characterizing thermodynamic efficiency of oxygen consumption). State 1 corresponded to the respiration of mitochondria oxidizing endogenous substrates (basal respiration, $V_{1}$ ). State 2 corresponded to the respiration in the presence of the substrate added $\left(V_{2}\right)$. The rate of mitochondrial respiration corresponding to State $3\left(V_{3}\right)$ was recorded after addition of $180 \mu \mathrm{M}$ ADP. State $4\left(V_{4}\right)$ corresponded to the respiration after ADP depletion. Oxygen consumption in each metabolic state was measured following $5 \mathrm{~min}$ after the corresponding additions (substrates or ADP) have been made. We expressed the mitochondrial respiration rates in the presence of the quinones as percentage of the control value $\left(V_{1}^{n}, V_{2}^{n}, V_{3}^{n}\right)$. We also used the parameter $<V>=\left(V_{1}^{n}+V_{2}^{n}\right) / 2$ removed by averaging the basal and substrate-dependent respiration rates.

\section{Cell culture}

C6 glioma cells were cultured in Dulbecco's modified Eagle's medium (DMEM, Sigma Chemical Co., St. Louis, MO, USA) supplemented with $10 \%$ fetal bovine serum and gentamicin $(50 \mu \mathrm{g} / \mathrm{ml})$. The cultures were incubated at $37{ }^{\circ} \mathrm{C}$ in a humidified $5 \% \mathrm{CO}_{2}$ and $95 \%$ air atmosphere. After the cells reached $70 \%$ confluency, they were harvested, washed 
with Hepes buffer (131 mM NaCl, $5 \mathrm{mM} \mathrm{KCl}, 1.3 \mathrm{mM} \mathrm{CaCl}_{2}, 1.3 \mathrm{mM} \mathrm{MgSO} 4,6 \mathrm{mM}$ glucose, $20 \mathrm{mM}$ Hepes, $\mathrm{pH} 7.3$ ), resuspended up to a final density of $1 \times 10^{6}$ cells $/ \mathrm{ml}$ and used for experiments.

\section{Mitochondrial membrane potential}

The mitochondrial membrane potential $(\Delta \psi)$ of isolated mitochondria was measured in the medium (0.2 M sucrose, $20 \mathrm{mM}$ Tris- $\mathrm{HCl}, 2.5 \mathrm{mM} \mathrm{MgSO}_{4}, 2.5 \mathrm{mM} \mathrm{KH}_{2} \mathrm{PO}_{4}, 20 \mathrm{mM} \mathrm{KCl}$, $1 \mathrm{mM}$ EDTA, pH 7.2) at $27^{\circ} \mathrm{C}$ fluorometrically using fluorescent probe safranin $\mathrm{O}(2)$. The excitation wavelength was $495 \mathrm{~nm}$ (slit $3 \mathrm{~nm}$ ) and the emission wavelength was $586 \mathrm{~nm}$ (slit $3 \mathrm{~nm}$ ) (an SM 2203 spectrofluorimeter, Solar, Belarus), the dye concentration was $8 \mu \mathrm{M}$ and the mitochondrial protein concentration was $0.3 \mathrm{mg} / \mathrm{ml}$. Isolated mitochondria were added to the media containing respiratory substrate $(5 \mathrm{mM}$ succinate or $5 \mathrm{mM}$ L-glutamate and $2 \mathrm{mM}$ malate mixture). At the end of the measurements, uncoupler FCCP $(0.5 \mu \mathrm{M})$ was added to the mitochondria to achieve a complete depolarization. The membrane potential values $(\mathrm{mV})$ were determined using a calibration plot, which represented the dependence of the safranin $\mathrm{O}$ fluorescence intensity on the membrane potential value, according to the Nernst equation:

$$
\Delta \psi=60 \log \frac{\left[\mathrm{K}^{+}\right]_{\text {out }}}{\left[\mathrm{K}^{+}\right]_{\text {in }}}(\mathrm{mV}),
$$

where $\left[\mathrm{K}^{+}\right]_{\text {in }}$ is the intramitochondrial potassium concentration $(120 \mathrm{mM})$ and $\left[\mathrm{K}^{+}\right]_{\text {out }}$ is the extramitochondrial potassium concentration in the media that varies from 0 to $20 \mathrm{mM}$ in the presence of ionophore valinomycin $(0.28 \mu \mathrm{M})(36)$.

Mitochondrial energization in intact C6 glioma cells was evaluated using mitochondrial membrane potential sensitive carbocyanine dye JC-1 (5,5',6,6'-tetrachloro-1,1',3,3'tetraethyl-benzamidazolocarbocyanine iodide) (44). This lipophilic and cationic dye accumulates within mitochondria driven by the negative membrane potential and forms concentration-dependent J-aggregates. When excited at $490 \mathrm{~nm}$, the monomeric form of the dye emits at $527 \mathrm{~nm}$, while the J-aggregates show maximum emission at $590 \mathrm{~nm}$. Briefly, $1 \times 10^{6}$ cells were treated with quinones for $20 \mathrm{~min}$, washed with Hepes buffer, and incubated in the presence of $1 \mu \mathrm{M} \mathrm{JC}-1$ for $15 \mathrm{~min}$. After washing cells twice again with Hepes buffer, JC-1-related fluorescence was analyzed in a spectrofluorimeter (SM2203, Solar, Belarus).

\section{ROS generation in mitochondria}

The mitochondrial ROS generation after addition of quinones $(1-10 \mu \mathrm{M})$ in the presence or absence of rotenone $(1 \mu \mathrm{M})$ was estimated fluorometrically using fluorescent probe $\mathrm{H}_{2} \mathrm{DCF}$ DA and Glu+Mal as respiratory substrate. $\mathrm{H}_{2}$ DCF-DA was de-esterified preliminarily by incubation with $0.1 \mathrm{M} \mathrm{NaOH}$ for 30 min to form non-fluorescent $\mathrm{H}_{2} \mathrm{DCF}$ (8). The $\mathrm{H}_{2} \mathrm{DCF}$ solution was adjusted to $\mathrm{pH} 7.4$ by addition of $0.1 \mathrm{M} \mathrm{HCl}$. The stock concentration of the $\mathrm{H}_{2}$ DCF was $100 \mu \mathrm{M}$. Ten microliters of the prepared $\mathrm{H}_{2}$ DCF solution was added to $1 \mathrm{ml}$ of the mitochondrial suspension $(0.3 \mathrm{mg}$ protein $/ \mathrm{ml})$. The extramitochondrial ROS were measured by oxidation of $\mathrm{H}_{2} \mathrm{DCF}$ to fluorescent $2^{\prime}, 7^{\prime}$-dichlorofluorescein (DCF) $(22,56)$. The experiments were carried out at $37^{\circ} \mathrm{C}$ and $\mathrm{pH} 7.4$. The DCF fluorescence intensity $\left(\lambda_{\mathrm{ex}}=\right.$ $488 \mathrm{~nm}, \lambda_{\mathrm{em}}=530 \mathrm{~nm}$ ) was recorded by a spectrofluorimeter for $40 \mathrm{~min}$.

For studying of mitochondrial superoxide production in C6 cells, MitoSOX Red [fluorogenic dye specifically targeted to mitochondria in live cells (45)] was added to $1.25-\mu \mathrm{M}$ final concentration for $20 \mathrm{~min}$, after which the cells were washed twice with Hepes 
buffer and analyzed. Fluorescence intensity of MitoSOX $\left(\lambda_{\text {ex }}=510 \mathrm{~nm}, \lambda_{\mathrm{em}}=580 \mathrm{~nm}\right)$ was measured using a spectrofluorimeter at $0,10,20,30$, and 40 min intervals after quinone addition to cell suspension.

\section{Statistical analysis}

The results were expressed as the means of four to five replicates \pm SD and the statistical analysis was conducted using the analysis of variance. We used the standard unpaired Student's $t$-test and nonparametric Spearman's rank correlation for the comparison of the raw and transformed data showing no departures from normality (according to Shapiro-Wilk's test). $p<0.05$ was taken to indicate statistical significance. Calculations were performed using the statistical software packages StatSoft Statistica 6.0 and GraphPad Prism 4.0. Correlation analysis was performed utilizing the GraphPad Prism 4.0. The fluorescent kinetic curves were typical for three to four independent experiments performed.

\section{Results}

Effects of quinones on the respiratory activity of mitochondria

We examined the respiratory activities of isolated rat liver mitochondria exposed to quinones of different chemical structures (Fig. 1). As Tables II and III show, the rate of basal oxygen consumption $\left(V_{1}\right)$, in the case of Glu+Mal as a respiratory substrate, increased twofold in the presence of DTBBQ, coenzyme $\mathrm{Q}_{0}$, and menadione $(10 \mu \mathrm{M})$. Simultaneously, DTBBQ decreased the ADP-stimulated respiration $V_{3}$ by $30 \%(p<0.05)$ without significant changes in the respiration rates $V_{2}$ and $V_{4}$. Coenzyme $\mathrm{Q}_{0}$ did not considerably influence the respiratory

Table II. Respiratory activity of rat liver mitochondria on exposure to quinones $(10 \mu \mathrm{M})$ during Glu+Mal oxidation

\begin{tabular}{|l|c|c|c|c|c|}
\hline & \multicolumn{1}{|c|}{$\begin{array}{c}\text { Basal } \\
\text { respiration } \\
\text { rate }\left(V_{1}^{n}\right), \\
\%\end{array}$} & $\begin{array}{c}\text { Glu+Mal- } \\
\text { dependent } \\
\text { respiration } \\
\text { rate } \\
\left(V_{2}^{n}\right), \%\end{array}$ & $\begin{array}{c}\text { ADP- } \\
\text { stimulated } \\
\text { respiration } \\
\text { rate } \\
\left(V_{3}^{n}\right), \%\end{array}$ & $\begin{array}{c}\text { Respiration } \\
\text { rate after } \\
\text { ADP } \\
\text { consumption } \\
\left(V_{4}^{n}\right), \%\end{array}$ & $<\boldsymbol{V}^{\prime}=\left(\frac{\boldsymbol{V}_{1}^{n}+\boldsymbol{V}_{2}^{n}}{2}\right), \%$ \\
\hline Control & 100 & 100 & 100 & 100 & 100 \\
\hline BQ & $121 \pm 30$ & $104 \pm 23$ & $103 \pm 15$ & $94 \pm 31$ & 113 \\
\hline DTBBQ & $217 \pm 47^{*}$ & $90 \pm 14$ & $71 \pm 17 *$ & $95 \pm 20$ & 154 \\
\hline TMBQ & $135 \pm 46$ & $98 \pm 15$ & $102 \pm 10$ & $100 \pm 13$ & 117 \\
\hline Coenzyme $Q_{0}$ & $228 \pm 40^{*}$ & $77 \pm 30$ & $98 \pm 18$ & $100 \pm 10$ & 153 \\
\hline Coenzyme $Q_{10}$ & $128 \pm 38$ & $67 \pm 45$ & $94 \pm 21$ & $62 \pm 35^{*}$ & 97 \\
\hline Menadione & $232 \pm 47^{*}$ & $159 \pm 35^{*}$ & $111 \pm 27$ & $188 \pm 35^{*}$ & 195 \\
\hline Juglone & $65 \pm 20^{*}$ & $231 \pm 50^{*}$ & $79 \pm 10^{*}$ & $302 \pm 70^{*}$ & 148 \\
\hline Lawsone & $100 \pm 28$ & $67 \pm 34$ & $106 \pm 17$ & $63 \pm 23^{*}$ & 83 \\
\hline
\end{tabular}

*Statistically significant in comparison with control, $p<0.05$ 
Table III. ACR, RCR, and phosphorylation coefficient of rat liver mitochondria on exposure to quinones $(10 \mu \mathrm{M})$ during Glu + Mal oxidation

\begin{tabular}{|l|c|c|c|}
\hline & ACR $\left(\boldsymbol{V}_{\mathbf{3}} / \boldsymbol{V}_{\mathbf{2}}\right)$ & RCR $\left(\boldsymbol{V}_{\mathbf{3}} / \boldsymbol{V}_{\mathbf{4}}\right)$ & ADP/O \\
\hline Control & $4.3 \pm 1.1$ & $4.7 \pm 1.8$ & $2.7 \pm 0.4$ \\
\hline BQ & $4.1 \pm 1.5$ & $3.1 \pm 1.7$ & $2.1 \pm 0.8$ \\
\hline DTBBQ & $3.7 \pm 1.2$ & $2.7 \pm 1.0^{*}$ & $2.5 \pm 0.7$ \\
\hline TMBQ & $4.4 \pm 1.5$ & $3.6 \pm 0.5$ & $2.6 \pm 0.3$ \\
\hline Coenzyme $\mathrm{Q}_{0}$ & $6.6 \pm 1.1$ & $4.2 \pm 1.0$ & $3.0 \pm 0.7$ \\
\hline Coenzyme $\mathrm{Q}_{10}$ & $7.3 \pm 1.2^{*}$ & $6.3 \pm 0.8^{*}$ & $3.0 \pm 0.7$ \\
\hline Menadione & $2.8 \pm 0.2^{*}$ & $2.7 \pm 0.7^{*}$ & $2.3 \pm 0.4$ \\
\hline Juglone & $1.8 \pm 0.5^{*}$ & $1.3 \pm 0.4^{*}$ & $1.2 \pm 0.8^{*}$ \\
\hline Lawsone & $8.3 \pm 1.0^{*}$ & $7.2 \pm 1.0^{*}$ & $2.5 \pm 0.5$ \\
\hline
\end{tabular}

*Statistically significant in comparison with control, $p<0.05$

rates $V_{2}, V_{3}$, and $V_{4}$, whereas menadione significantly enhanced the respiration rate $V_{2}$ (by $60 \%, p<0.05$ ) and $V_{4}$ (by $90 \%, p<0.05$ ), unchanging the rate $V_{3}$. Juglone caused a decrease of the $V_{1}$ (by $35 \%, p<0.05$ ) and $V_{3}$ respiration rates, but significantly elevated the rates of Glu+Mal-stimulated respiration $\left(V_{2}\right.$, by $\left.130 \%, p<0.05\right)$ and respiration after ADP depletion $\left(V_{4}\right.$, by $\left.200 \%, p<0.05\right)$. BQ and TMBQ did not change the respiration significantly. Coenzyme $\mathrm{Q}_{10}$ and lawsone decreased the respiration rate $V_{4}$ (by $40 \%$, $p<0.05)$ and did not change the rates $V_{1}, V_{2}$, and $V_{3}$. The values for ACR and RCR were considerably increased in the presence of coenzyme $\mathrm{Q}_{10}$ and lawsone $(1.3-1.7$-fold, $p<0.05)$ (Table III). The phosphorylation coefficient (ADP/O) was unchanged after addition of these quinones. During mitochondrial exposure to menadione or juglone, the ACR was observed to decrease 1.5- and 2.4-fold ( $p<0.05$ ), respectively, whereas the RCR was found to diminish 1.7-fold $(p<0.05)$ for DTBBQ or menadione and 3.6-fold $(p<0.05)$ for juglone. Juglone most pronouncedly lowered the ACR, RCR, and ADP/O values. These data indicate that juglone is an effective uncoupling agent of mitochondrial respiration.

\section{Quinone restoration of mitochondrial respiration during inhibition}

It was shown earlier that menadione, idebenone, coenzyme $\mathrm{Q}_{0}$, and coenzyme $\mathrm{Q}_{1}$, but not coenzyme $\mathrm{Q}_{10}$, partially restored mitochondrial activity under conditions of impaired Complex I function $(23,37)$. In our experiments, addition of amytal $(10 \mu \mathrm{M})$, Complex I inhibitor, caused significant impairment in mitochondrial respiratory activity (Tables IV and V). TMBQ and menadione stimulated the basal, Glu+Mal-dependent, and ADP-stimulated respirations and increased the values of $\mathrm{ACR}, \mathrm{RCR}$, and $\mathrm{ADP} / \mathrm{O}(p<0.05)$, restoring functions of the mitochondrial ETC during inhibition of Complex I by amytal. The protective effect of coenzyme $\mathrm{Q}_{0}$ was less pronounced (Tables IV and V). The other quinones studied did not show any significant preventing effect during Complex I inhibition (data not shown). As Tables IV and V indicate, the addition of two-electron quinone reduction inhibitor (mitochondrial DT-diaphorase inhibitor), dicumarol, to mitochondria during inhibition of Complex I prevented restoration of mitochondrial respiratory activity by menadione 
Table $I V$. Effect of quinones $(10 \mu \mathrm{M})$ on Glu + Mal-stimulated respiratory activity of rat liver mitochondria during inhibition of Complex I by amytal $(10 \mu \mathrm{M})$ in the absence and the presence of dicumarol

\begin{tabular}{|c|c|c|c|c|}
\hline & $\begin{array}{c}\text { Basal } \\
\text { respiration } \\
\text { rate }\left(V_{1}^{n}\right), \\
\% \\
\end{array}$ & $\begin{array}{c}\text { Glu + Mal- } \\
\text { dependent } \\
\text { respiration } \\
\text { rate }\left(V_{2}^{n}\right), \% \\
\end{array}$ & $\begin{array}{c}\text { ADP- } \\
\text { stimulated } \\
\text { respiration } \\
\text { rate }\left(V_{3}^{n}\right), \% \\
\end{array}$ & $\begin{array}{c}\text { Respiration } \\
\text { rate after ADP } \\
\text { consumption } \\
\left(V_{4}^{n}\right), \% \\
\end{array}$ \\
\hline Control & 100 & 100 & 100 & 100 \\
\hline Amytal & $81 \pm 33$ & $66 \pm 22$ & $40 \pm 22$ & $94 \pm 37$ \\
\hline Amytal + TMBQ & $136 \pm 15^{*}$ & $106 \pm 15^{*}$ & $66 \pm 13$ & $97 \pm 9$ \\
\hline Amytal + menadione & $132 \pm 10^{*}$ & $140 \pm 29 *$ & $91 \pm 12^{*}$ & $177 \pm 17 *$ \\
\hline Amytal + coenzyme $\mathrm{Q}_{0}$ & $120 \pm 10$ & $99 \pm 16$ & $36 \pm 16$ & $104 \pm 31$ \\
\hline \multicolumn{5}{|c|}{ Addition of dicumarol $(10 \mu \mathrm{M})$} \\
\hline Amytal + dicumarol & $155 \pm 11$ & $158 \pm 60$ & $60 \pm 10$ & $168 \pm 34$ \\
\hline $\begin{array}{l}\text { Amytal + dicumarol + } \\
\text { TMBQ }\end{array}$ & $110 \pm 15$ & $200 \pm 74$ & $54 \pm 25$ & $169 \pm 45$ \\
\hline $\begin{array}{l}\text { Amytal + dicumarol+ } \\
\text { menadione }\end{array}$ & $180 \pm 15$ & $249 \pm 60^{*}$ & $87 \pm 22$ & $332 \pm 70^{*}$ \\
\hline $\begin{array}{l}\text { Amytal + dicumarol + } \\
\text { coenzyme } \mathrm{Q}_{0}\end{array}$ & $165 \pm 12$ & $160 \pm 70$ & $70 \pm 15$ & $150 \pm 30$ \\
\hline
\end{tabular}

*Statistically significant in comparison with corresponding control without quinone, $p<0.05$

(partially), coenzyme $\mathrm{Q}_{0}$ and TMBQ (completely). Thus, the restoration of the respiratory chain functions by quinones was due to their conversions to the phenolic form.

\section{Effect of quinones on mitochondrial membrane potential}

We studied the effect of naphtho- and benzoquinones on the membrane potential of intact mitochondria and under Complex I inhibition. In our experiments, quinones, impairing mitochondria respiration, induced dissipation of the mitochondrial membrane potential. As Fig. 2 demonstrates, addition of juglone $(10 \mu \mathrm{M})$ caused a slow partial decrease of the membrane potential from $-200 \mathrm{mV}$ (for the control) to $-150 \mathrm{mV}$ within $10-15 \mathrm{~min}$ of Glu +Mal oxidation and did not affect the membrane potential during succinate oxidation (Fig. 3), which indicates quinone interaction with Complex I. The effect of DTBBQ on the membrane potential was less pronounced $(\Delta \psi$ was decreased by $12 \pm 3 \mathrm{mV}, p<0.05)$ during both $\mathrm{Glu}+\mathrm{Mal}$ and succinate oxidation (Figs 2 and 3). Figures 2 and 3 show that lawsone, menadione, coenzyme $\mathrm{Q}_{10}$, and TMBQ at a concentration of $10 \mu \mathrm{M}$ did not influence the membrane potential under Glu+Mal or succinate oxidation. At the same time, as shown in Fig. 4, menadione and TMBQ $(10 \mu \mathrm{M})$ restored the Glu+Mal-generated mitochondrial potential to $-(200 \pm 23) \mathrm{mV}$ (completely), or $-(150 \pm 15) \mathrm{mV}$ (partially), respectively, after rotenone addition. Rotenone dissipated Glu+Mal - but not succinate-generated membrane potential. The inhibitor was added after $200 \mathrm{~s}$ following the quinone injection (Figs 3 and 4). Surprisingly, lawsone also slightly prevented rotenone-induced potential loss (Fig. 4). Dicumarol inhibited quinone restoration of the mitochondrial membrane potential dissipated 
Table $V$. Effects of quinones $(10 \mu \mathrm{M})$ on ACR, RCR, and phosphorylation coefficient of rat liver mitochondria during inhibition of Complex I by amytal $(10 \mu \mathrm{M})$ in the absence and the presence of dicumarol; Glu + Mal is respiratory substrate

\begin{tabular}{|l|c|c|c|}
\hline & ACR $\left(\boldsymbol{V}_{\mathbf{3}} / \boldsymbol{V}_{\mathbf{2}}\right)$ & $\mathbf{R C R}\left(\boldsymbol{V}_{\mathbf{3}} / \boldsymbol{V}_{\mathbf{4}}\right)$ & ADP/O \\
\hline Control & $4.3 \pm 1.1$ & $4.7 \pm 1.8$ & $2.7 \pm 0.4$ \\
\hline Amytal & $1.7 \pm 0.5$ & $1.5 \pm 0.5$ & $1.7 \pm 0.7$ \\
\hline Amytal + TMBQ & $2.6 \pm 0.1^{*}$ & $2.7 \pm 0.4^{*}$ & $2.1 \pm 0.3^{*}$ \\
\hline Amytal + menadione & $2.6 \pm 0.3^{*}$ & $2.3 \pm 0.6$ & $2.9 \pm 0.5^{*}$ \\
\hline Amytal + coenzyme Q & $2.3 \pm 0.6$ & $1.9 \pm 0.4$ & $2.1 \pm 0.6$ \\
\hline $\begin{array}{c}\text { Addition of dicumarol }(10 \mu \mathrm{M}) \\
\text { Amytal + dicumarol }\end{array}$ & $1.8 \pm 0.5$ & $1.6 \pm 1.0$ & $0.9 \pm 0.6$ \\
\hline $\begin{array}{c}\text { Amytal + dicumarol + } \\
\text { TMBQ }\end{array}$ & $2.3 \pm 0.6$ & $2.0 \pm 0.7$ & $1.2 \pm 0.6$ \\
\hline $\begin{array}{c}\text { Amytal + dicumarol + } \\
\text { menadione }\end{array}$ & $1.7 \pm 0.3$ & $1.3 \pm 0.6$ & $2.0 \pm 0.7^{*}$ \\
\hline $\begin{array}{c}\text { Amytal + dicumarol + } \\
\text { coenzyme Q }\end{array}$ & $2.0 \pm 0.6$ & $1.7 \pm 0.5$ & $1.0 \pm 0.7$ \\
\hline
\end{tabular}

* Statistically significant in comparison with corresponding control without quinone, $p<0.05$

by rotenone: completely in the case of TMBQ and partially in the case of menadione. BQ did not affect the membrane potential in the absence of rotenone but enhanced the inhibitory effect of rotenone during Glu+Mal oxidation (Fig. 4). Similarly, juglone was found to enhance the action of rotenone, increasing the rate of mitochondrial membrane potential loss

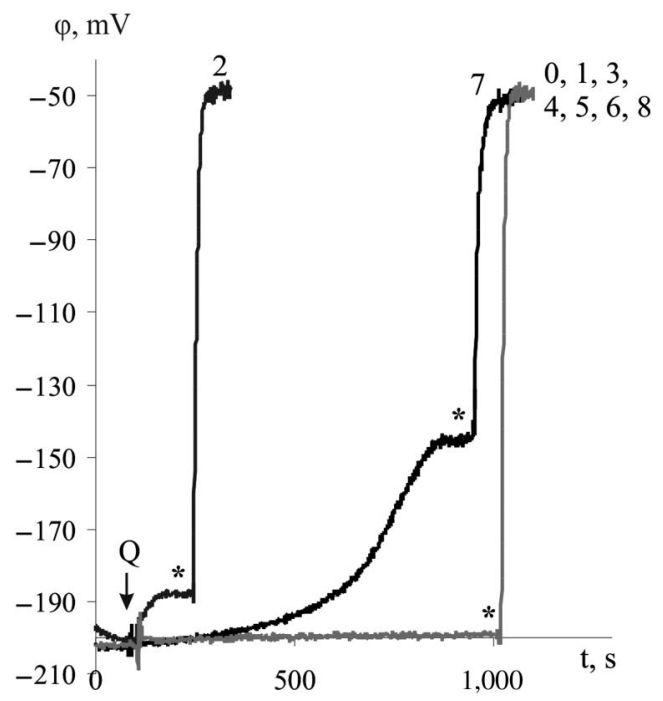

Fig. 2. Mitochondrial membrane potential in rat liver mitochondria exposed to $10 \mu \mathrm{M}$ quinones with Glu + Mal as respiratory substrate: 0 , control; $1, \mathrm{BQ} ; 2$, DTBBQ; 3, TMBQ; 4, coenzyme $\mathrm{Q}_{0} ; 5$, coenzyme $\mathrm{Q}_{10} ; 6$, menadione; 7, juglone; 8, lawsone. Q and * designate moments of quinone and FCCP addition, respectively 

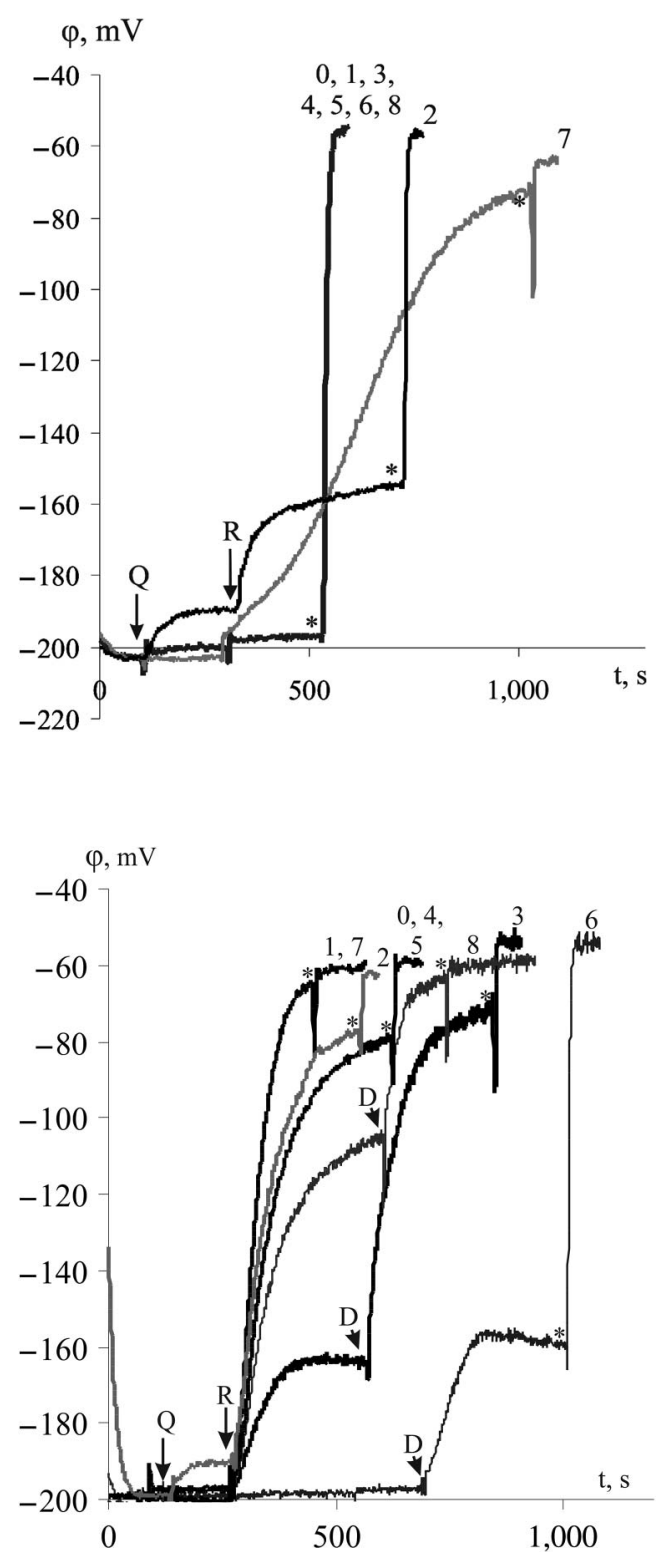

Fig. 3. Mitochondrial membrane potential in rat liver mitochondria exposed to $10 \mu \mathrm{M}$ quinones and rotenone $(1 \mu \mathrm{M})$ with succinate as respiratory substrate: 0 , control; 1, BQ; 2, DTBBQ; 3, TMBQ; 4, coenzyme $\mathrm{Q}_{0} ; 5$, coenzyme $\mathrm{Q}_{10} ; 6$, menadione; 7, juglone; 8 , lawsone. Q, R, and * designate moments of quinone, rotenone and FCCP addition, respectively

Fig. 4. Mitochondrial membrane potential in rat liver mitochondria exposed to $10 \mu \mathrm{M}$ quinones, rotenone

$(1 \mu \mathrm{M})$ and dicumarol $(1 \mu \mathrm{M})$ with Glu+Mal as respiratory substrate: 0 , control; $1, \mathrm{BQ} ; 2$, DTBBQ; 3, TMBQ; 4, coenzyme $\mathrm{Q}_{0} ; 5$, coenzyme $\mathrm{Q}_{10} ; 6$, menadione; 7, juglone; 8, lawsone. Q, R, D, and * designate moments of quinone, rotenone, dicumarol, and FCCP addition, respectively

(Fig. 4). DTBBQ, coenzyme $\mathrm{Q}_{0}$, and coenzyme $\mathrm{Q}_{10}$ did not prevent the inhibitory effect of rotenone. Figure 3 shows that rotenone did not change the membrane potential in succinateoxidizing mitochondria but considerably dissipated the succinate-generated potential in the presence of juglone or DTBBQ. The combined action of DTBBQ and rotenone decreased the membrane potential during succinate oxidation by $45 \pm 9 \mathrm{mV}, p<0.05$, and the combined action of juglone and rotenone induced a complete loss of the potential. We suggested that juglone or DTBBQ catalyzed the reverse electron flow from Complex II to Complex I and, thus, in this way participated in the generation of the succinate-dependent membrane potential. 


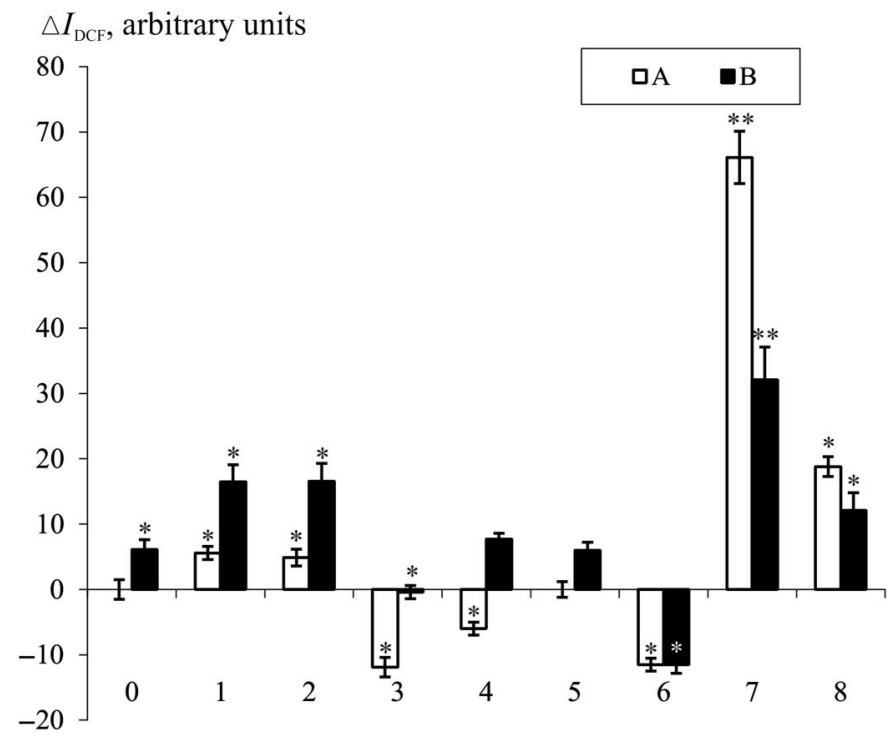

Fig. 5. The change of mitochondrial ROS production under exposure to quinones in the absence (A) and in the presence of $1 \mu \mathrm{M}$ rotenone (B) under Glu + Mal oxidation: 0, control; 1, BQ $(1 \mu \mathrm{M}) ; 2$, DTBBQ $(10 \mu \mathrm{M}) ; 3$, TMBQ $(10 \mu \mathrm{M})$; 4, coenzyme $\mathrm{Q}_{0}(10 \mu \mathrm{M}) ; 5$, coenzyme $\mathrm{Q}_{10}(10 \mu \mathrm{M}) ; 6$, menadione $(10 \mu \mathrm{M}) ; 7$, juglone $(1 \mu \mathrm{M}) ; 8$, lawsone $(10 \mu \mathrm{M})$. Zero level corresponds to ROS production by intact mitochondria; *Statistically significant in comparison with the corresponding control without quinone, $p<0.05$. **Statistically significant in comparison with the corresponding control without quinone, $p<0.01$

\section{Effect of quinones on the ROS generation in mitochondria}

To elucidate the role of redox processes in the quinone effects on mitochondrial functions, we determined the quinone-induced ROS release to the extramitochondrial medium (Fig. 5). It was found that $10-\mu \mathrm{M}$ DTBBQ and lawsone or $1-\mu \mathrm{M}$ BQ or juglone increased ROS generation in rat liver mitochondria oxidizing Complex I substrate Glu+Mal. Maximal amount of ROS was registered under the action of juglone. On the contrary, a decrease of ROS production in mitochondria was revealed on TMBQ, menadione, and coenzyme $\mathrm{Q}_{0}$ addition. Coenzyme $\mathrm{Q}_{10}$ did not affect ROS generation.

The inhibition of Complex I by rotenone led to the increase in Glu+Mal-stimulated ROS production in control mitochondria and in the presence of DTBBQ and BQ. However, rotenone partially inhibited juglone- and lawsone-induced ROS generation and prevented inhibition of ROS production by TMBQ and coenzyme $\mathrm{Q}_{0}$. Complex I inhibition did not influence mitochondrial ROS production in the presence of menadione and coenzyme $\mathrm{Q}_{10}$.

Effect of quinones on mitochondrial membrane potential and ROS generation in C6 cells Figure 6 shows the alteration of mitochondrial membrane potential in glioma cell exposed to $10-\mu \mathrm{M}$ quinones for $30 \mathrm{~min}$. It is seen that in contrast to isolated mitochondria, a decrease in mitochondrial potential in intact cells was detected during treatment with all the quinones, except for coenzyme $\mathrm{Q}_{10}$. It was found that juglone addition led to practically complete mitochondrial depolarization, while $\mathrm{BQ}$ and menadione induced 50\% loss of mitochondrial membrane potential. In cells exposed to TMBQ, coenzyme $\mathrm{Q}_{0}$, and lawsone, the mitochondrial membrane potential was $20 \%-30 \%$ less than in the control cells. At the same time, 


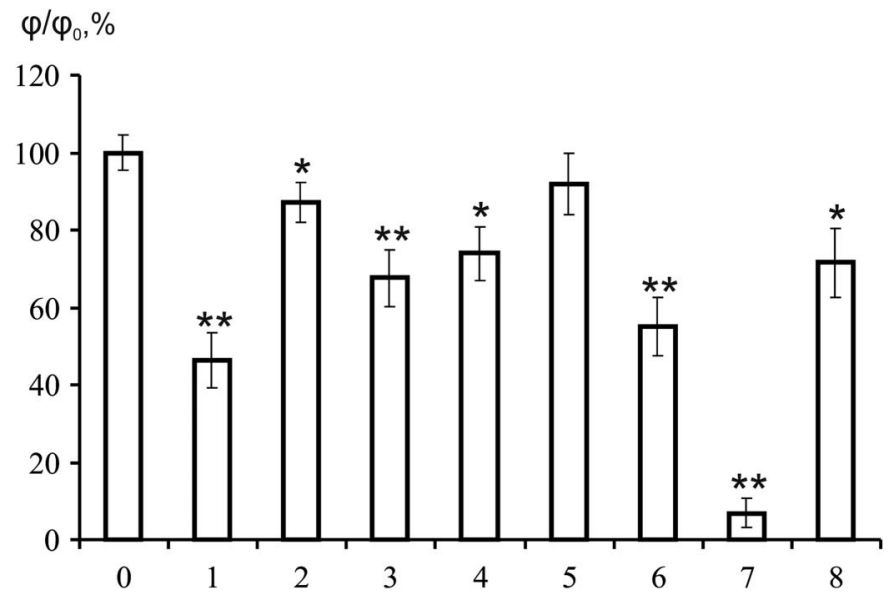

Fig. 6. Mitochondrial membrane potential of $\mathrm{C} 6$ glioma cell exposed to $10 \mu \mathrm{M}$ quinones for $30 \mathrm{~min}$ : 0 , control; 1, BQ; 2, DTBBQ; 3, TMBQ; 4, coenzyme $\mathrm{Q}_{0} ;$ 5, coenzyme $\mathrm{Q}_{10} ; 6$, menadione; 7, juglone; 8, lawsone. Each value of mitochondrial membrane potential has been divided on corresponding value $\varphi_{0}$ of the potential in control samples and represented as a percentage. Zero level corresponds to uncoupling at FCCP addition. *Statistically significant in comparison with the corresponding control without quinone, $p<0.05$. **Statistically significant in comparison with the corresponding control without quinone, $p<0.01$

DTBBQ changed the potential weakly: the mitochondrial membrane potential in treated cells was $(87 \pm 5) \%$ relative to control.

Figure 7 demonstrates the mitochondrial superoxide generation levels in C6 cells exposed to $10 \mu \mathrm{M}$ quinones. It was shown that, similarly to isolated mitochondria, $\mathrm{BQ}$, DTBBQ, juglone, and lawsone induced additional $\mathrm{O}_{2}{ }^{\cdot-}$ production, while TMBQ, coenzyme

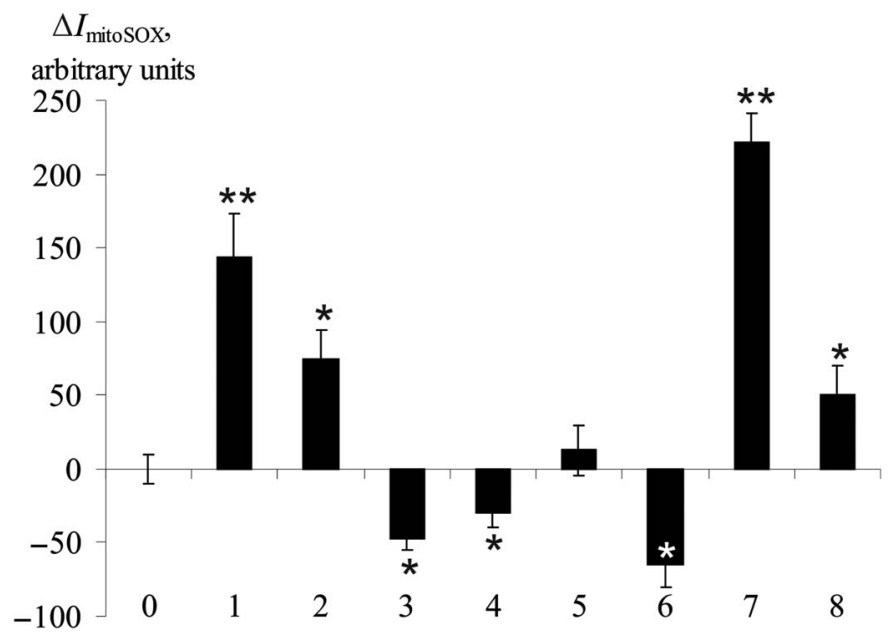

Fig. 7. The change of mitochondrial superoxide anion radicals production in $\mathrm{C} 6$ glioma cells under exposure to $10 \mu \mathrm{M}$ quinones: 0 , control; 1, BQ; 2, DTBBQ; 3, TMBQ; 4, coenzyme $\mathrm{Q}_{0} ; 5$, coenzyme $\mathrm{Q}_{10} ; 6$, menadione; 7, juglone; 8, lawsone. Zero level corresponds to mitochondrial superoxide production by intact cells. *Statistically significant in comparison with the corresponding control without quinone, $p<0.05$. ** Statistically significant in comparison with the corresponding control without quinone, $p<0.01$ 
$\mathrm{Q}_{0}$, and menadione decreased $\mathrm{O}_{2}{ }^{--}$yield in mitochondria of intact cells. However, it is noteworthy that coenzyme $\mathrm{Q}_{0}$, menadione, and juglone induced significant cytosolic ROS production in cells (data not shown).

\section{Discussion}

It is well known that quinones considerably influence mitochondrial function $(5,20,21,26$, 51). Reduction of quinone by mitochondrial ETC complexes or DT-diaphorase can result in formation of semi- or dihydro- forms of quinone molecules. Electrochemical behavior of semi- or dihydroquinones (quinoles) can be different $(6,38)$. Reduced quinones can autoxidize, inducing ROS generation, or can transfer electrons to Complexes II, III, or IV, shunting ETC functions, changing redox balance in mitochondria, and working as potent antioxidants $(13,23,37,46,47,49,55)$.

In our experiments, exogenous quinones considerably changed mitochondrial respiration, membrane potential, and ROS generation for isolated mitochondria or whole cells. Enhanced oxygen consumption by mitochondria (the increased $V_{1}$ and $V_{2}$ rates), which we registered in the presence of some quinones, can be due to both activation of substrate oxidation and ROS production in the quinone redox cycle with $\mathrm{O}_{2}$. Earlier, Briere et al. (5) suggested that either idebenone or decylubiquinone enhanced succinate-dependent oxygen uptake in mitochondria due to significant stimulation of an electron flow from succinate to oxygen and activation of succinate dehydrogenase. On the other hand, Kelso et al. (28) showed that quinone derivative mitoQ significantly stimulated coupled glutamate/ malate-dependent respiration of mitochondria by increasing a proton leak through inner membrane. The authors concluded that mitoQ was reduced and oxidized (recycled) by the mitochondrial respiratory complexes (28).

Depending on the structure and properties, the quinones affected mitochondrial ETC functions in different ways: the more lipophilic quinones studied, juglone, menadione, and DTBBQ, with $E_{1 / 2}$ in the range from -99 to $-260 \mathrm{mV}$, disturbed mitochondrial ETC functions, decreasing the RCR and ACR coefficients (uncoupling effect); menadione, TMBQ, coenzyme $\mathrm{Q}_{0}$ decreased ROS generation; menadione, TMBQ restored the ETC functions during inhibition of Complex I; juglone, lawsone, DTBBQ, and BQ induced ROS generation; lawsone and coenzyme $\mathrm{Q}_{10}$ enhanced the RCR and $\mathrm{ACR}$ values; and juglone and DTBBQ catalyzed the reverse electron flow from Complex II to Complex I.

We evaluated the relationships between the solubility of the quinones (Table I) and their effects on mitochondrial ETC functions (Fig. 8). A correlation analysis was performed, disregarding coenzyme $\mathrm{Q}_{10}$ which did not affect mitochondrial function. This may be because coenzyme $\mathrm{Q}_{10}$ is a native component of the ETC and the endogenous coenzyme $\mathrm{Q}_{10}$ has already been in excess. From the other hand, it is known that exogenous quinones can be reduced both at physiologic sites of Complex I ETC and non-physiologic ones, which are accessible for hydrophilic compounds (31). Due to the high hydrophobicity (the $\log P$ for coenzyme $\mathrm{Q}_{10}$ is almost an order of magnitude higher than the $\log P$ for the other quinones), coenzyme $\mathrm{Q}_{10}$ can be beyond the solubility range in which the correlation is significant. A correlation between the ACR and RCR values (Table III) and the quinone lipophilic properties $[\log P$, the partition coefficient in octanol/water system for the quinones studied, (53)] was found. The nonparametric Spearman's rank correlation coefficients $\rho_{\mathrm{ACR}}$ and $\rho_{\mathrm{RCR}}$ were equal to -0.49 $\left(P_{\mathrm{ACR}}=0.045\right)$ and $-0.49\left(P_{\mathrm{RCR}}=0.049\right)$, respectively. However, the correlation between the $\mathrm{ADP} / \mathrm{O}\left(P_{\mathrm{ADP} / \mathrm{O}}=0.43\right)$ and the quinone lipophilic properties was not ascertained (Fig. 8). It 

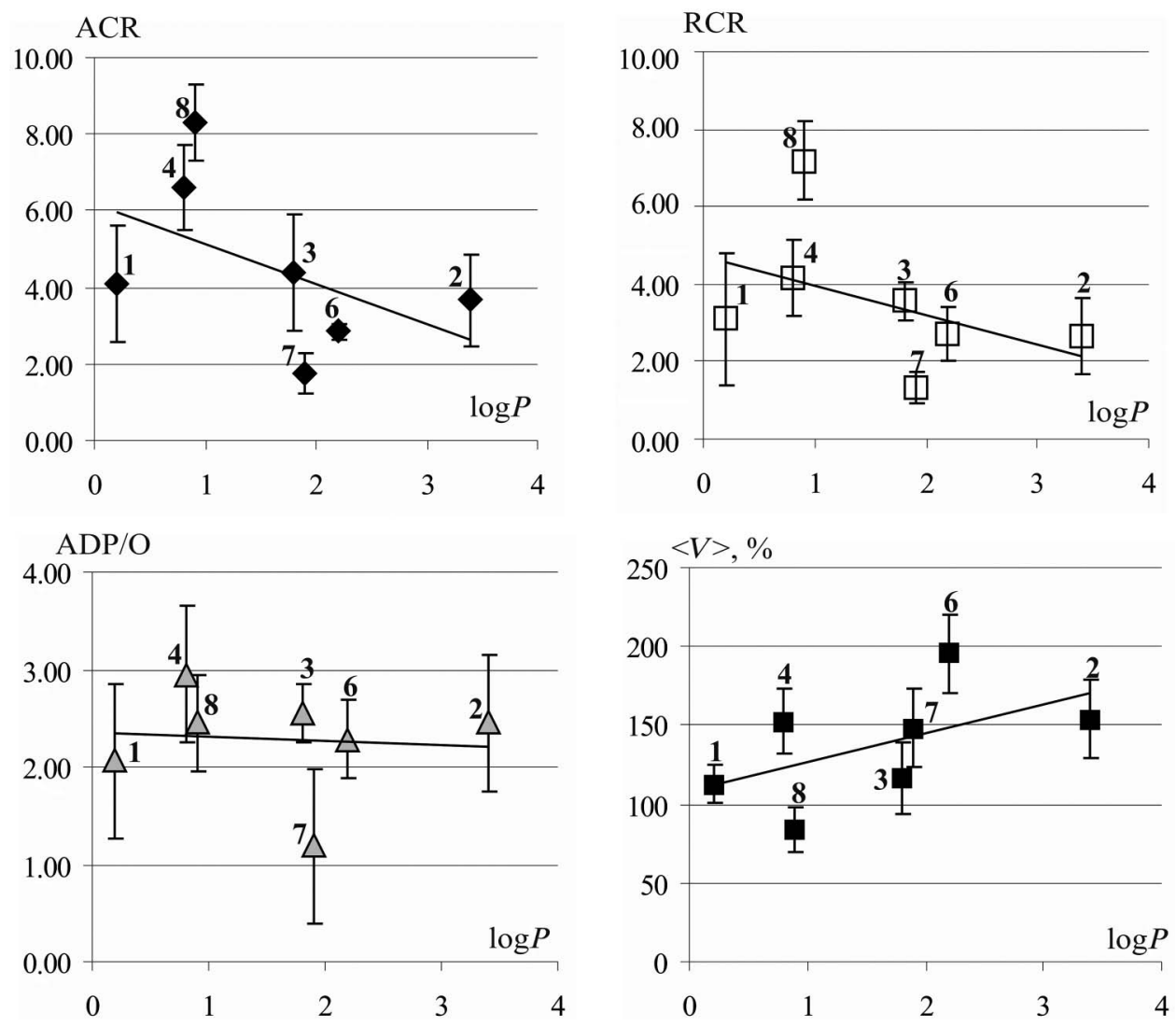

Fig. 8. Relationships between ACR, RCR, ADP/O, and $\langle V\rangle$ and quinone lipophilicity: 1, BQ; 2, DTBBQ; 3, TMBQ; 4, coenzyme $\mathrm{Q}_{0} ; 5$, coenzyme $\mathrm{Q}_{10} ; 6$, menadione; 7, juglone; 8, lawsone

was shown previously that less hydrophobic quinone derivatives poorly affected the electron flow from either NADH or succinate to oxygen in mitochondria (5).

It is known that exogenous quinones can both be oxidized and reduced by ETC complexes $(11,13,23,28,37,46,47,49,55)$. However, because they are not a primary substrate of these enzymes, the efficiency of oxidation/reduction has to be strongly dependent on the availability of primary substrates and "saturation" of electrons in the ETC (reduction states of components of ETC), just as the electron leak from ETC and superoxide production depend on the reduction states of ETC components and the proton gradient (25). In the absence of ADP, the mitochondrial membrane potential, stored as a proton gradient, is not consumed on ATP production and ETC is saturated, providing favorable conditions for reduction of exogenous quinones by ETC complexes, and, therefore, the greatest changes in oxygen consumption due to the reduction of quinones should be revealed for $V_{2}$ respiration rate. On the other hand, the oxidation of quinols will proceed most efficiently under conditions of lower ETC activity, namely, in the absence of respiratory substrates. The basal respiration rate $V_{1}$ is supported by a minimum of substrates that were preserved in mitochondria during isolation process. The greatest changes in oxygen consumption due to the oxidation of quinols should be registered for $V_{1}$ respiration rate. The efficiency of quinone 


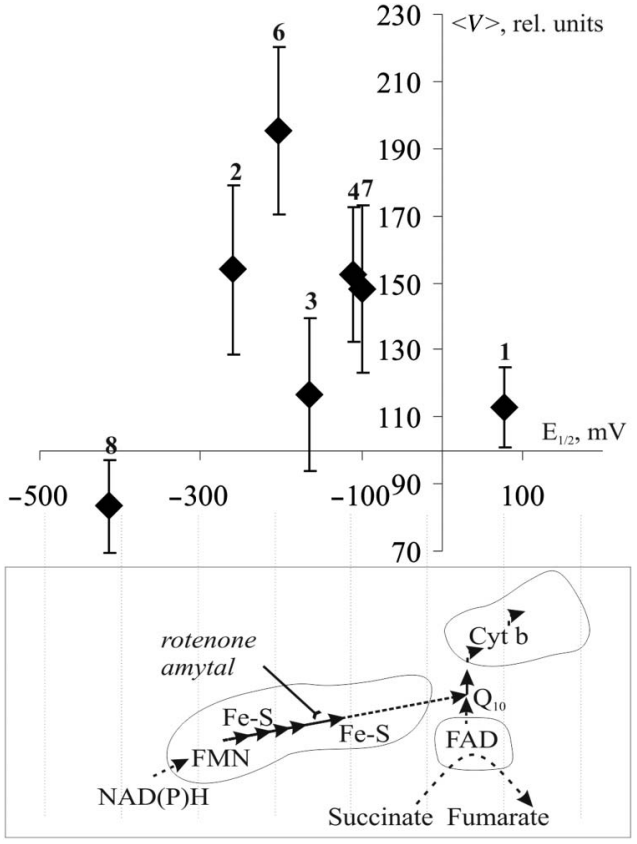

Fig. 9. Scheme for mitochondrial electron transfer with redox potential markers and relationship between $\langle V\rangle$ (efficiency of quinone-ETC interaction) and quinone one-electron reduction potential: 1, BQ; 2, DTBBQ; 3, TMBQ; 4, coenzyme $\mathrm{Q}_{0} ; 5$, coenzyme $\mathrm{Q}_{10} ; 6$, menadione; 7, juglone; 8 , lawsone

interaction with ETC depended on the processes of oxidation and reduction of quinones by ETC. Because of this, the total parameter $\left(V_{1}+V_{2}\right)$ has to be analyzed, taking both oxidation and reduction of quinones into consideration. Hence, $\langle V\rangle$ in the presence of quinones reflects their redox activity in the ETC.

We evaluated the relationships between quinone solubility and $\langle V\rangle$. A correlation between $\langle V\rangle$ and $\log P$ was found with the correlation coefficients $\rho_{\mathrm{V}}$ being equal to 0.44 $\left(P_{\mathrm{V}}=0.05\right)$. Thus, the efficiency of quinone-ETC interaction increases and the respiration parameters (ACR and RCR) decrease with the rise of quinone lipophilicity in the range of $\log P$ from 0.2 to 3.4. Figure 9 shows the dependence of $\langle V\rangle$ on the quinone one-electron redox potential and schematically represents the ETC, with the elements being located according to the reduction potential values. It is seen that the $\langle V\rangle$ increased and, respectively, the efficient quinone-ETC interfering occurred at the quinone redox potential values from -260 to $-99 \mathrm{mV}$. The potential values from $-320 \mathrm{mV}$ (for NADH) to $-36 \mathrm{mV}$ (for ubiquinone) correspond to Complex I, whereas the $E_{1 / 2}$ of DTBBQ $\left(E_{1 / 2}=-260 \mathrm{mV}\right)$ corresponds to the potential of the NADH: ubiquinone reductase initial site, while the $E_{1 / 2}$ of juglone $\left(E_{1 / 2}=-99 \mathrm{mV}\right)$ is close to the Q-reduction site of Complex I. It is known that rotenone interrupts electron transfer in Complex I, which causes a decrease of ROS generation by Complex III and an increase of ROS generation by Complex I (3, 12, 30, $32,33,48,54)$. Then, the ROS production by quinones, which are reduced in ETC before rotenone binding site of Complex I, would increase after Complex I inhibition and, vice versa, the ROS production by quinones, which are reduced at the Complex I ubiquinonebinding site that is located higher than the rotenone-binding site in ETC would be suppressed during rotenone treatment. Since DTBBQ-induced ROS generation increased while jugloneinduced ROS generation decreased during Complex I inhibition (Fig. 5), we have concluded that the most probable site of electron leak to DTBBQ is the Complex I flavin site, while sites 
of electron leak to juglone were likely to be localized at or close to the Q-reduction site of Complex I.

To explain the influence of rotenone (inhibitor of the Complex I) on the membrane potential of mitochondria energized by succinate (substrate of the Complex II) in the presence of juglone or DTBBQ, one can suggest that these quinones catalyze the reverse electron flow from Complex II to Complex I (rotenone completely inhibited succinate-generated potential in the presence of juglone, and partially - in the presence of DTBBQ). We assume that the reduction of juglone and DTBBQ by Complex I can alter the electron path between the ETC complexes, resulting in a reverse electron flow between Complexes I and II in succinateenergized mitochondria.

The leak of electrons from the ETC to juglone or DTBBQ produced semi- and dihydroquinones. Taking into consideration, the $E_{1 / 2}$, semi-quinone of juglone can readily react with molecular oxygen $(6,7)$, resulting in mitochondrial ROS generation (Fig. 5). Due to this, juglone and DTBBQ were more effective in disturbance of the respiratory parameters of mitochondria (Tables II and III). Quinone-induced oxidative stress in mitochondria participates in a slow decrease of the mitochondrial potential as well as in uncoupling of respiration and phosphorylation $(17,42)$.

Although menadione $\left(E_{1 / 2}=-203 \mathrm{mV}\right)$, TMBQ $\left(E_{1 / 2}=-165 \mathrm{mV}\right)$, and coenzyme $\mathrm{Q}_{0}$ $\left(E_{1 / 2}=-110 \mathrm{mV}\right)$ possess one-electron redox potential value lying in the range of Complex I potential values, the efficiencies of quinone-ETC interaction vary markedly for these quinones. On the other hand, menadione, TMBQ, and coenzyme $\mathrm{Q}_{0}$ decreased ROS production by mitochondria. According to Fig. 9, menadione is the most effective redox-active quinone that interferes with ETC. At the same time, menadione did not disrupt mitochondrial functioning as did juglone. On the contrary, in our experiments, menadione decreased mitochondrial ROS production and did not induce membrane potential loss. In accordance with our observations Chan et al. showed that coenzyme $\mathrm{Q}_{1}$ and menadione $(5 \mu \mathrm{mol} / \mathrm{l})$ considerably prevented rotenone-induced mitochondrial ROS production and rotenone cytotoxicity as well as restored mitochondrial membrane potential and ATP production in isolated hepatocytes (9). Coenzyme $\mathrm{Q}_{1} \mathrm{H}_{2}$, idebenone, and menadiol reduced by DT-diaphorase act as superoxide radicals scavengers $(9,23,50)$.

In addition, ROS production is the result of quinone autoxidation and it depends on the rate of this process. The rate of menadione autoxidation following its reduction by DTdiaphorase is 50 times less compared to juglone (7). Due to this fact, menadiol, in contrast to juglone, has time to transfer electrons to Complex III before it will be oxidized by molecular oxygen. Thus, menadiol oxidation in ETC resulted in a decrease of superoxide production and restoration of ETC functions during inhibition of Complex I.

Interestingly, TMBQ $\left(E_{1 / 2}=-165 \mathrm{mV}\right)$ per se did not affect mitochondrial activities during both Glu+Mal and succinate oxidation, but similarly to menadione, it was reduced by DT-diaphorase, inhibited mitochondria ROS generation and partially restored ETC functions during inhibition of Complex I (Table V and Fig. 4). Coenzyme $\mathrm{Q}_{0}$ is distinguished from TMBQ by change of methyl groups to methoxy ones. Meanwhile, opposite to TMBQ, mitochondria exposure to coenzyme $\mathrm{Q}_{0}$ led to an increase in the basal respiration rate (possibly due to the electron leak from ETC to quinones), but it did not feed electrons to Complex III. It was shown (10) that coenzyme $\mathrm{Q}_{1}$ was optimal for preventing rotenonemediated cell death as compared to both $\mathrm{CoQ}_{0}$ and $\mathrm{CoQ}_{2}$. Therefore, coenzyme $\mathrm{Q}_{0}$ can be considered as a possible electron carrier along the ETC during inhibition of Complex I 
functions (37). Nevertheless, it is not an effective agent for restoration of mitochondrial functions possibly due to its low lipophilicity. Comparing all the quinone compounds, which can restore mitochondrial functioning under conditions of impaired mitochondrial Complex I function, notably coenzyme $\mathrm{Q}_{1}(\log P=2.8)$, duroquinone $\left(\log P=2.45, E_{1 / 2}=-260 \mathrm{mV}\right)$, idebenone $(\log P=4.3)$, menadione $\left(\log P=2.2, E_{1 / 2}=-203 \mathrm{mV}\right)$, and TMBQ $(\log P=1.8$, $\left.E_{1 / 2}=-165 \mathrm{mV}\right)$, it can be concluded that the therapeutic agents should be sufficiently lipophilic (with the $\log P$ in the range from 1.8 to 4.3 ) and have a one-electron reduction potential value in the range of $(-165 /-260) \mathrm{mV}$. It should be noted that the quinone electron and steric properties depended on the aromatic ring substituents, e.g., TMBQ, CoQ ${ }_{0}, \mathrm{CoQ}_{1}$, menadione, and idebenone having at least one methyl group in the quinoid ring restored mitochondrial functions, and TMBQ, CoQ 0 , and menadione inhibited ROS generation in mitochondria.

BQ $\left(E_{1 / 2}=+78 \mathrm{mV}\right)$ did not participate in the redox cycle and it had no effect on the mitochondrial respiration parameters and potential (Table II and Fig. 3). However, BQ potentiated the action of rotenone, i.e., Complex I activity was decreased, which is in agreement with other data (41), and induced ROS production by mitochondria. BQ-induced ROS generation may be connected with a quinone reaction with reduced glutathione (the reaction rate constant is $2 \mu \mathrm{M}^{-1} \mathrm{~s}^{-1}$ ) (6). It should be noted that juglone and benzoquinone, as compared to the other quinones studied, are the most effective arylating agents. Due to this fact, juglone and benzoquinone adverse effects on mitochondrial function can result from direct arylation of some essential $\mathrm{SH}-$ groups of ETC complexes.

Lawsone $\left(E_{1 / 2}=-415 \mathrm{mV}\right)$ increased the ACR and RCR coefficients, but did not affect the mitochondrial potential. Nevertheless, the increase of ROS production was detected under exposure to lawsone. Since lawsone did not interfere with ETC, the lawsone-induced ROS generation was probably due to lawsone reduction by DT-diaphorase followed by its autoxidation and hydrogen peroxide generation in the extramitochondrial medium.

The effects of coenzyme $Q_{10}$ on the mitochondrial respiration parameters or the values for membrane potential were not pronounced. Probably because coenzyme $Q_{10}$ is a native component of the ETC and it has already been in excess in the mitochondria.

On the other hand, the quinones could regulate mitochondrial permeability transition and in this way influence the mitochondrial respiration and potential. It was shown earlier by Petronilli et al. (42) that menadione $(25 \mu \mathrm{M})$ stimulated mitochondrial permeability transition pore opening by shifting its gating potential, and mitochondrial $\mathrm{SH}-$ groups modification completely prevented menadione-induced pore opening. Similarly, Palmeira and Wallace have shown that 1,4-naphthoquinone, 2-methyl-1,4-naphthoquinone (menadione), and 2,3dimethoxy-1,4-naphthoquinone elicited an increase in the gating potential of permeability. Benzoquinone, on the other hand, prevented induction of the mitochondrial permeability transition by all the three redox-cycling naphthoquinones due to the electrophilic, sulfhydrylarylating reactivity of benzoquinone (41).

It should be noted that in cellular systems, the mechanisms of quinone effects on the mitochondrial potential are primary mediated by cytosolic ROS generation rather than by direct action on mitochondrial components. Modification of cellular homeostasis, alteration of membrane lipid organization, and induction of oxidative stress and/or increase of $\mathrm{Ca}^{2+}$ intracellular level can contribute to quinone-induced mitochondrial effects on the cellular level $(20,26)$. 


\section{Conclusions}

Exogenous quinones differing in chemical structure, redox potential, and lipophilic properties are capable of effective participation in mitochondrial processes, interacting with the respiratory chain complexes, and controlling electron transfer, affect respiratory parameters of mitochondria, redox homeostasis in mitochondrial compartments, dissipate mitochondrial membrane potential, influence (decrease or enhance) ROS generation, and restore electron flow during ETC inhibition by rotenone in isolated mitochondria and intact cells.

DTBBQ and juglone either are inhibitors of ETC, or they accept electrons from ETC and reduce $\mathrm{O}_{2}$ to superoxide (or both). TMBQ and menadione can participate in ETC as electron carriers, while they are reduced by DT diaphorase or accept electrons from Complex I. While reduced, they also scavenge ROS instead of generating it.

The respiration parameters characterizing the effect of quinones on mitochondrial physiology correlated with quinone lipophilicity and depended on the redox potential and chemical structure.

\section{Acknowledgements}

This work was supported by the grants of Belarusian State University and Yanka Kupala State University of Grodno and the grant (no. M13-102) of the Belarusian Republican Foundation for Fundamental Research.

\section{Conflict of interest}

The authors declare that they have no conflict of interest.

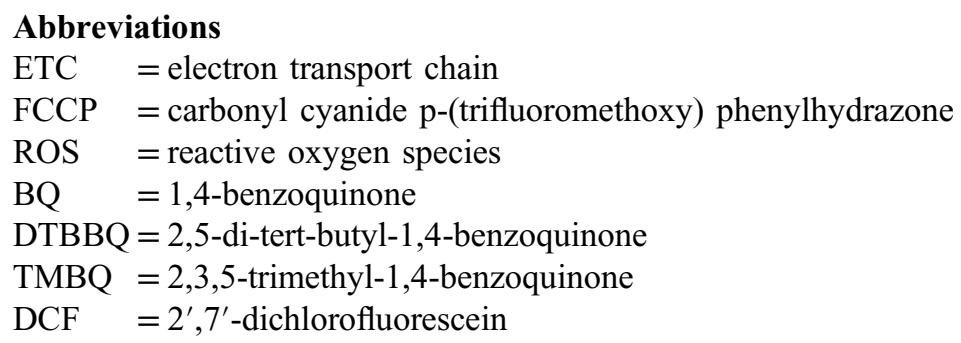

\section{REFERENCES}

1. Adams R, Bachmann WE, Fieser LF, Blatt AH, Johnson JR (1948): Organic Reactions. John Wiley, New York, NY, USA

2. Akerman KE: Qualitative measurements of the mitochondrial membrane potential in situ in Ehrlich ascites tumour cells using the safranin method. Biochim. Biophys. Acta 546, 341-347 (1979)

3. Andreyev AY, Kushnareva YE, Starkov AA: Mitochondrial metabolism of reactive oxygen species. Biochemistry (Mosc.) 70, 200-214 (2005)

4. Brand MD: The sites and topology of mitochondrial superoxide production. Exp. Gerontol. 45, 466-472 (2010)

5. Briere JJ, Schlemmer D, Chretien D, Rustin P: Quinone analogues regulate mitochondrial substrate competitive oxidation. Biochem. Biophys. Res. Commun. 316, 1138-1142 (2004)

6. Brunmark A, Cadenas E: Redox and addition chemistry of quinoid compounds and its biological implications. Free Radic. Biol. Med. 7, 435-477 (1989)

7. Buffinton GD, Ollinger K, Brunmark A, Cadenas E: DT-diaphorase-catalysed reduction of 1, 4-naphthoquinone derivatives and glutathionyl-quinone conjugates. Effect of substituents on autoxidation rates. Biochem. J. 257, 561-571 (1989) 
8. Cathcart R, Schwiers E, Ames BN: Detection of picomole levels of hydroperoxides using a fluorescent dichlorofluorescein assay. Anal. Biochem. 134, 111-116 (1983)

9. Chan TS, Teng S, Wilson JX, Galati G, Khan S, O’Brien PJ: Coenzyme Q cytoprotective mechanisms for mitochondrial Complex I cytopathies involves $\mathrm{NAD}(\mathrm{P}) \mathrm{H}$ : quinone oxidoreductase 1 (NQO1). Free Radic. Res. 36, 421-427 (2002)

10. Chan TS, Wilson JX, O’Brien PJ: Coenzyme Q cytoprotective mechanisms. Methods Enzymol. 382, 89-104 (2004)

11. Chen M, Liu B, Gu L, Zhu Q: The effect of ring substituents on the mechanism of interaction of exogenous quinones with the mitochondrial respiratory chain. Biochim. Biophys. Acta 851, 469-474 (1986)

12. Chen Q, Vazquez EJ, Moghaddas S, Hoppel CL, Lesnafsky EJ: Production of reactive oxygen species by mitochondria. Central role of complex III. J. Biol. Chem. 278, 36027-36031 (2003)

13. Connover TE, Ernster L: DT diaphorase. II. Relation to respiratory chain of intact mitochondria. Biochim. Biophys. Acta 58, 189-192 (1962)

14. Deller S, Macheroux P, Sollner S: Flavin-dependent quinone reductases. Cell. Mol. Life Sci. 65, 141-160 (2008)

15. de Vries S, Berden JA, Slater EC: Properties of a semiquinone anion located in the $\mathrm{QH}_{2}: \mathrm{cytochrome} \mathrm{c}$ oxidoreductase segment of the mitochondrial respiratory chain. FEBS Lett. 122, 143-148 (1980)

16. Dremza IK, Lapshina EA, Kujawa J, Zavodnik IB: Oxygen-related processes in red blood cells exposed to tertbutyl hydroperoxide. Redox Rep. 11, 185-192 (2006)

17. Fagian M, Pereira-da-Silva L, Martins IS, Vercesi AE: Membrane protein thiol cross-linking associated with the permeabilization of the inner mitochondrial membrane by $\mathrm{Ca}^{2+}$ plus prooxidants. J. Biol. Chem. 265, 19551960 (1990)

18. Fieser LF, Fieser M (1968): Reagents for Organic Synthesis. John Wiley, New York, NY, USA

19. Flaig W, Ploetz T, Biergans H: Information on humic acids. Report on the formation and reactions of some hydroxyquinones. Justus Liebigs Ann. Chem. 597, 196-213 (1956)

20. Gerasimenko JV, Gerasimenko OV, Palejwala A, Tepikin AV, Petersen OH, Watson AJ: Menadione-induced apoptosis: roles of cytosolic $\mathrm{Ca}^{2+}$ elevations and the mitochondrial permeability transition pore. J. Cell. Sci. 115, 485-497 (2002)

21. Giorgio V, Petronilli V, Ghelli A, Carelli V, Rugolo M, Lenaz G, Bernardi P: The effects of idebenone on mitochondrial bioenergetics. Biochim. Biophys. Acta 1817, 363-369 (2012)

22. Gomes A, Fernandes E, Lima JL: Fluorescence probes used for detection of reactive oxygen species. J. Biochem. Biophys. Methods 65, 45-80 (2005)

23. Haefeli RH, Erb M, Gemperli AC, Robay D, Courdier FI, Anklin C, Dallmann R, Gueven N: NQO1-Dependent redox cycling of idebenone: effects on cellular redox potential and energy levels. PLoS One 6, e17963 (2011)

24. Henry TR, Wallace KB: Differential mechanisms of induction of the mitochondrial permeability transition by quinones of varying chemical reactivities. Toxicol. Appl. Pharmacol. 134, 195-203 (1995)

25. Jastroch M, Divakaruni AS, Mookerjee S, Treberg JR, Brand MD: Mitochondrial proton and electron leaks. Essays Biochem. 47, 53-67 (2010)

26. Ji Y, Qu Z, Zou X: Juglone-induced apoptosis in human gastric cancer SGC-7901 cells via the mitochondrial pathway. Exp. Toxicol. Pathol. 63, 69-78 (2011)

27. Johnson D, Lardy HA: Isolation of liver or kidney mitochondria. Methods Enzymol. 10, 94-96 (1967)

28. Kelso GF, Porteous CM, Coulter CV, Hughes G, Porteous WK, Ledgerwood EC, Smith RA, Murphy MP: Selective targeting of a redox-active ubiquinone to mitochondria within cells: antioxidant and antiapoptotic properties. J. Biol. Chem. 276, 4588-4596 (2001)

29. Kruglov AG, Subbotina KB, Saris NE: Redox-cycling compounds can cause the permeabilization of mitochondrial membranes by mechanisms other than ROS production. Free Radic. Biol. Med. 44, 646-656 (2008)

30. Lambert AJ, Brand MD: Inhibitors of quinone-binding site allow rapid superoxide production from mitochondrial NADH: ubiquinone oxidoreductase. J. Biol. Chem. 279, 39414-39420 (2004)

31. Lenaz G: Quinone specificity of Complex I. Biochim. Biophys. Acta 1364, 207-221 (1998)

32. Lenaz G: The mitochondrial production of reactive oxygen species: mechanisms and implications in human pathology. IUBMB Life 52, 159-164 (2001)

33. Li N, Ragheb K, Lawler G, Sturgis J, Rajwa B, Melendez JA, Robinson JP: Mitochondrial complex I inhibitor rotenone induces apoptosis through enhancing mitochondrial reactive oxygen species production. J. Biol. Chem. 278, 8516-8525 (2003)

34. Lowry OH, Rosebrough NJ, Farr AL, Randall RJ: Protein measurement with the Folin phenol reagent. J. Biol. Chem. 193, 265-275 (1951) 
35. Marchi S, Giorgi C, Suski JM, Agnoletto C, Bononi A, Bonora M, DeMarchi E, Missiroli S, Patergnani S, Poletti F, Rimessi A, Duszynski J, Wieckowski MR, Pinton P: Mitochondria-ROS crosstalk in the control of cell death and aging. J. Signal Transduct. 2012, 329635 (2012)

36. Moore AL, Bonner WD: Measurements of membrane potentials in plant mitochondria with the safranin method. Plant Physiol. 70, 1271-1276 (1982)

37. Motovilov K, Yaguzhinsky L: Inhibitors of succinate dehydrogenase and complex III promote respiration of liver mitochondria under conditions of functioning DT-diaphorase. Biochim. Biophys. Acta 1777, S98-S99 (2008)

38. Mukherjee T (2001): Radiation chemistry of quinones. In: Radiation Chemistry: Present Status and Future Trends, eds Jonah CD, Rao BSM, Elsevier Science, Amsterdam, The Netherlands, pp. 287-317

39. O’Brien PJ: Molecular mechanisms of quinone cytotoxicity. Chem. Biol. Interact. 80, 1-41 (1991)

40. Ollinger K, Kågedal K: Induction of apoptosis by redox-cycling quinones. Subcell. Biochem. 36, 151-170 (2002)

41. Palmeira CM, Wallace KB: Benzoquinone inhibits the voltage-dependent induction of the mitochondrial permeability transition caused by redox-cycling naphthoquinones. Toxicol. Appl. Pharmacol. 143, 338-347 (1997)

42. Petronilli V, Costantini P, Scorrano L, Colonna R, Passamonti S, Bernardi P: The voltage sensor of the mitochondrial permeability transition pore is tuned by oxidation-reduction state of vicinal thiols. Increase of the gating potential by oxidants and its reversal by reducing agents. J. Biol. Chem. 269, 16638-16642 (1994)

43. Powis G, Appel PL: Relationship of the single-electron reduction potential of quinones to their reduction by flavoproteins. Biochem. Pharmacol. 29, 2567-2572 (1980)

44. Reers M, Smith TW, Chen LB: J-aggregate formation of a carbocyanine as a quantitative fluorescent indicator of membrane potential. Biochemistry 30, 4480-4486 (1991)

45. Robinson KM, Janes MS, Pehar M, Monette JS, Ross MF, Hagen TM, Murphy MP, Beckman JS: Elective fluorescent imaging of superoxide in vivo using ethidium-based probes. Proc. Natl. Acad. Sci. U S A 103, 15038-15043 (2006)

46. Ruzicka FJ, Crane FL: Four quinone reduction sites in the NADH-dehydrogenase complex. Biochem. Biophys. Res. Commun. 38, 249-254 (1970)

47. Ruzicka FJ, Crane FL: Quinone interaction with the respiratory chain-linked NADH-dehydrogenase of beef heart mitochondria. Juglone reductase activity. Biochim. Biophys. Acta 223, 71-85 (1970)

48. Selivanov VA, Votyakova TV, Pivtoraiko VN, Zeak J, Sukhomlin T, Trucco M, Roca J, Cascante M: Reactive oxygen species production by forward and reverse electron fluxes in the mitochondrial respiratory chain. PLoS Comput. Biol. 7, e1001115 (2011)

49. Shadyro OI, Glushonok GK, Glushonok TG, Edimecheva IP, Moroz AG, Sosnovskaya AA, Yurkova IL, Polozov GI: Quinones as free-radical fragmentation inhibitors in biologically important molecules. Free Radic. Res. 36, 859-867 (2002)

50. Siegel G, Reigan P, Ross D (2008): One- and two-electron-mediated reduction of quinones: enzymology and toxicological implication. In: Advances in Bioactivation Research, ed Elfarra AA, Springer, New York, NY, USA, pp. 1-29

51. Siraki AG, Chan TS, O'Brien P: Application of quantitative structure-toxicity relationships for the comparison of the cytotoxicity of 14 p-benzoquinone congeners in primary cultured rat hepatocytes versus PC12 cells. Toxicol. Sci. 81, 148-159 (2004)

52. Swallow AJ (1982): Physical chemistry of semiquinone. In: Function of Quinones in Energy Conserving Systems, ed Trumpower B, Academic Press, New York, NY, USA, p. 71

53. The PubChem Project: Databases. Available at: http://pubchem.ncbi.nlm.nih.gov. Accessed 1 May 2013

54. Treberg JR, Quinlan CL, Brand MD: Evidence for two sites of superoxide production by mitochondrial NADHubiquinone oxidoreductase (complex I). J. Biol. Chem. 286, 27103-27110 (2011)

55. Vervoort LM, Randen JE, Thijssen HH: The potent antioxidant activity of the vitamin K cycle in microsomal lipid peroxidation. Biochem. Pharmacol. 54, 871-876 (1997)

56. Wrona M, Wardman P: Properties of the radical intermediate obtained on oxidation of $2^{\prime}, 7^{\prime}$-dichlorodihydrofluorescein, a probe for oxidative stress. Free Radic. Biol. Med. 41, 657-667 (2006) 